SLAC-PUB-8165

May, 1999

\title{
Long-term Operational Experience with the Barrel CRID at SLD*
}

\author{
J.Va'vra \\ Representing the CRID Collaboration \\ Stanford Linear Accelerator Center, Stanford University, Stanford, CA 94309. U.S.A.
}

Abstract

The Barrel CRID detector has been operating successfully at SLD for the past seven years. It is an important tool for SLD physics analyses. The long-term operational experience with this device is described.

Invited talk at the Third International Workshop on Ring Imaging Cherenkov Detectors, Ein-Gedi, Dead Sea, Israel, November 15-20, 1998

* Work supported by The Department of Energy, contract DEAC03-76SF00515. 


\section{INTRODUCTION}

In the mid-1980s, it was far from clear that one could operate a large system such as CRID for five to ten years. Singleelectron detection on such a large scale and UV operation is difficult. There were many variables we worried about in the early days. Ten years after finishing the construction, the major issues we struggled with were: (a) breaking of $7 \mu \mathrm{m}$ dia. wires, (b) purity of fluorocarbons, (c) occasional background from SLC, (d) sulfur in ethane, (e) recovery of amplifier to very large pulses, and ( $f$ ) contamination of optics in monochromators. Nevertheless, the CRID's particle identification capability is impressive, as can be seen in Fig.1; CRID identifies pions between $350 \mathrm{MeV} / \mathrm{c}$ and $35 \mathrm{GeV} / \mathrm{c}$, kaons between $750 \mathrm{MeV} / \mathrm{c}$ and $35 \mathrm{GeV} / \mathrm{c}$, and protons between $750 \mathrm{MeV} / \mathrm{c}$ and $45 \mathrm{GeV} / \mathrm{c}$. A summary of CRID's physics accomplishments are described in Ref.1.

The concept of the CRID detection scheme at SLD benefited from pioneering work by J. Seguinot, T. Ypsilantis [2], T. Ekelof, and many others. The overall design is similar to that of the DELPHI RICH [3]. The early R\&D developments of the Barrel CRID are summarized in Ref.4, and its electrostatic design is described in Ref.5. The detector's main operating features, and early CRID performance, are described in Refs.6.

The CRID includes 40 TPC's, 40 liquid radiator trays containing liquid $\mathrm{C}_{6} \mathrm{~F}_{14}$, a vessel containing a gas radiator composed of $87 \% \mathrm{C}_{5} \mathrm{~F}_{12}+13 \% \mathrm{~N}_{2}$, and a system of 400 spherical mirrors [7]. The photoelectron drifts to a wire chamber where its position is reconstructed using a combination of drift time ( $\mathrm{z}$-coordinate), wire address ( $\mathrm{x}$-coordinate) and charge division ( $\mathrm{y}$-coordinate) with a precision of $\sim 1 \times 1 \times 2 \mathrm{~mm}$. The TPC gas is $\mathrm{C}_{2} \mathrm{H}_{6}+\mathrm{TMAE}(\sim 0.1 \%)$. The maximum drift length is $1.2 \mathrm{~m}$, and a maximum operating voltage of $55 \mathrm{kV}$, which gives a drift field of $400 \mathrm{~V} / \mathrm{cm}$. The system operates at $35^{\circ} \mathrm{C}$ with the TMAE bubbler temperature set at $26-27^{\circ} \mathrm{C}$, and has 3720 anode wires, 7440 amplifiers, 64000 field shaping electrodes, and 6520 corona-preventing field wires [5,6].

\section{SYSTEM PERFORMANCE}

\section{A. Electrostatic design and high voltage}

To define the drift field in the TPC's, CRID used simple field cage wire geometry (see Fig.2) rather than a volume degrader. Our experience with the high voltage system is excellent proving that it is not necessary to build a volume degrader for voltages up to $60 \mathrm{kV}$ and distances of $8-10 \mathrm{~cm}$ to ground, provided that a gas, such as $\mathrm{C}_{5} \mathrm{~F}_{12}$, with good dielectric properties fills the volume. However, the field cage wires must have no defects, as they could create corona even in $\mathrm{C}_{5} \mathrm{~F}_{12}$ gas (Ref. 8 and Fig.3). An example of such a possible defect could be a broken etched array wire (such an accident has never happened fortunately). In the carly days, the DELPHI group argued that the field cage wires must be located precisely to ensure good field inside the TPC. We stressed, instead, that it is important to control a possible corona, and therefore, the field cage wires must be thick. We worried that any corona on the field cage wires might cause very large photon backgrounds in nearby TPCs. This did not occur during the entire ten-year period, mainly because of our conservative design for the electric gradient on the field cage wires $\left(E_{\text {wire }}<\right.$ $25 \mathrm{kV} / \mathrm{cm}$ ). The angular resolution of the Cherenkov gas rings near the expected value proves that it was not critical to have the field cage wires located precisely.

We had good operational experiences with: (a) high voltage cables, (b) all power supplies, (c) resistors used for TPC field grading, and (d) spark gaps used to protect them in case of a spark. In retrospect, it was vital that all detector HV controls were individual (Ref.8 and Fig.4); this made it possible to treat any defect with a high degree of flexibility. The cathode current per TPC was monitored to a nA sensitivity; a HV detector cathode trip level per TPC was set to $300 \mathrm{nA}$ to be sensitive to the Malter current as much as possible. In retrospect, this limit should have been even lower.

\section{B. The Single Electron Detector \\ 1) General Performance}


In retrospect, when I compare CRID with other designs I have tried, the CRID detector works very well from the point of view of quenching and overall electrostatics. A small wire diameter causes a nonlinear amplification at large gas gains, which reduces the sparking rate. Indeed, during the SLD operation, the CRID detector behaved very well as far as the sparking compared to other gaseous detectors in SLD.

The single-electron detector [9] is based on single stage MWPC wire amplification with an average total gas gain of $\sim 2-3 \times 10^{5}$ [10]. We are running as low a gas gain as possible because of a respect for the wire ageing, the Malter current, and the cross-talk (the low gas gain affects the efficiency and the charge division). The present CRID operating point corresponds to a single electron efficiency of about $85-90 \%$ [11]. To limit the avalanche photons entering the TPC drift volume, a system of Cu-Be blinds was constructed. The measured rate of after-pulses per avalanche caused by the secondary avalanche photons is $<1 \%$ at our operating point. The Cu-Be blind structure gives a factor of 7-8 reduction in the rate of after-pulsing. In order to have good electron transmission to the anode wire, the ratio of the drift field within the detector to that in the TPC is maintained at a value close to six. This makes the structure relatively insensitive to misalignments.

\section{2) Charge Division Performance}

Initial experience and the very good charge division resolution (the resolution along the wire length), $\sigma_{2} / 1 \sim 0.7 \%$, obtained in early R\&D tests $[4,10]$, led to the choice of $7 \mu \mathrm{m}$ carbon wire. As a result of wire ageing arguments, the wire gas gain is set as low value as possible. This is the dominant factor in defining the observed charge division resolution, $\sigma_{z} / 1 \sim 2.5 \%$ [12]. However, a calculation [13] shows that the $\sim 2.5 \%$ charge division resolution contributes little to the final angular resolution per point in the liquid rings (gas rings are affected even less).

3) Wire Ageing

The TMAE molecule is susceptible to polymerization. The prediction from the early R\&D ageing tests [14] is that already a modest detector current of about 5-10nA would cause a 50\% gain drop in about 1-2 years. The R\&D data also predicted that the smaller the wire diameter, the larger the ageing rate, and the TMAE wire ageing rate is larger than that of the TEA molecule [15].

Figure 5(a) shows the wire ageing at SLD and indicates a decrease in gain of about 30-40\% between 1993 and 1998; this result is consistent with the R\&D data (assuming that an average current at SLD is $2-4 n A$ per detector). From pulse height distribution measurements, it is estimated that a $30 \%$ average gain decrease reduces the detection efficiency by about $5 \%$. The rate of wire ageing was studied by monitoring the average single electron pulse height using UV fibers.

To regenerate the wire gain, we have shown, in R\&D tests, that we can either wash the detectors with alcohol, or evaporate the deposits by passing a small $(\sim 10 \mathrm{~mA})$ current which heats the carbon wires to a temperature above $300^{\circ} \mathrm{C}$. However, after $\sim 7$ years of running, there is strong evidence that the wires have weakened to the point that they may be unable to tolerate this current without running a substantial risk of breakage. Tests with a detector that was removed from SLD recently indicate that such a procedure would be too risky. Therefore, the only option available is to clean the wires by washing the whole anode plane in ethanol. This was done to some detectors being repaired in the lab. Figure 5(b) indeed shows that there is an improvement in the relative average pulse height after such washing. However, this improvement seems to be temporary.

\section{4) Malter Effect}

It is well known that a strong radiation source can easily excite large, steady, self-sustaining Malter currents (typically few tens of nA, up to several $\mu \mathrm{A}$ ) [16], if the cathode surface is covered by an insulator. However, it was shown recently [17] that the Malter effect can "manifest" itself as sporadic emissions of charge in a form of single electrons.

It was already known during an early CRID R\&D period that one could excite the self-sustaining currents, typically several hundred $\mathrm{nA}$, in a CRID detector by a UV lamp shining a large photon flux. Therefore, we were actively searching for some 
evidence of such currents during the regular SLD running. The first evidence was found when one detector developed such currents while running with the gating. When the gating is on the positive ions drift from the anode wires to the gating wires, rather than into the TPC volume. Apparently, some insulating region on a wire of one detector caused the charge buildup, and subsequent, steady self-sustaining currents. The next evidence came from the UV fiber operation. During the period from 1991 to 1993, our UV fiber trigger rate was set to the $120 \mathrm{~Hz}$ SLC repetition rate. This caused local rates on some anode wires to be as high as $10 \mathrm{~Hz}$ per $\mathrm{cm}$ of wire length, and caused damage, probably in the form of an insulating film, to cathodes corresponding to wire locations aligned with the UV fiber fiducials. It was discovered [17] that the damage would show up as bursts of charge in a form of single electrons every 15-20 minutes (see Fig.6). After the problem was discovered, the UV fiber trigger rate was reduced by a factor of 2000 , and this yielded a reduction of the burst rate by more than a factor of 20 . However, since then, these wires cannot take a very high UV fiber rate. The total charge dose from the UV fiber source before the first bursts were discovered was estimated to be only about $2 \times 10^{-7} \mathrm{C} / \mathrm{cm}$, which is an exceedingly small amount. Of course, the subsequent burst charges increased the total charge dose rapidly. So far, during hadronic trigger running, this has not caused a problem due to sufficiently low charge density. However, it is obviously a concern for times when the SLC background is very large. A similar concern may come from background muons aligned with the TPC axis.

\section{5) Wire Breaking}

In retrospect, the initial good experience with the $7 \mu \mathrm{m}$ diameter wires is a classical example of not catching problems occurring at a rate of less than 1:1000 during R\&D stages. These wires are definitely too fragile, and we break a wire every 1-1.5 months on average, and this creates geometrical inefficiencies at a level of 5-10\%. We try to repair detectors with broken wires during runs in order to minimize their impact. It is not clear why such breakage occurs, but we think it is due to material fatigue resulting from the total accumulated dose. This dose can be enhanced by excessive SLC noise during some periods, due to background muons depositing charge axially in the TPC ( $>10000 \mathrm{e}$ per track), or due to the charge created by bursts related to the Malter effect. In retrospect, a choice of an anode wire diameter between 20 and $33 \mu \mathrm{m}$ would have been better.

\section{B. Electronics Performance}

We placed the amplifiers, analog storage (HAMU), ADC's, and control and multiplexing circuitry on the detector [18]. Only a few serial optical fibers lead to the Fastbus located in the electronics control room. Although this concept is very elegant, it took a considerable amount of time to debug it (and many climbing trips to the SLD magnet). Typically, we have $\sim 1 \%$ dead electronics channels, randomly distributed at any given time during the run. From time to time, some other larger segments fail; for example, an ADC board, and this causes an additional 1-2\% dead channels, which tend to be clustered.

The amplifier charge gain is about $\sim 2.7 \mu \mathrm{V} /$ electron with a shaping time of about $65 \mathrm{~ns}$. In retrospect, the shaping time was chosen correctly; by reducing it to $\sim 20 \mathrm{~ns}$, the pulse amplitude would be decreased by a factor of four, which is equivalent to a cathode voltage increase of $\sim 150 \mathrm{Volts}$; this would cause already difficulties to operate detectors in a long run. To limit heating problems, the amplifiers are water cooled, and all components except the first JFET use pulsed power. The analog storage needs to be calibratcd cvery few days; the amplifier gain is calibrated once per run.

The amplifier-detector combination has a cross-talk at a level of $+1.1 \%$ for the first neighbor and about $-0.25 \%$ for a distant neighbor [19]. This may seem small, but a typical charged track deposits about 1000 electrons in the TPC; the cross-talk problem becomes significant [20]. The amplifier recovery from such charge deposits creates spurious pulses, and also affects the measurement of real pulses. This is a complication for CRID analysis in the core of a jet, where many tracks overlap; for example, it causes a typical loss of $10-20 \%$ of the photoelectrons per liquid ring. In retrospect, it was a mistake to make the TPC so thick $(8-10 \mathrm{~cm})$, which creates large $\mathrm{dE} / \mathrm{dx}$ deposits. 


\section{TPC Gas}

The $\mathrm{C}_{2} \mathrm{H}_{6}$ gas ${ }^{1}$ is cleaned in the standard way [21], i.e., we use mechanical filters, ${ }^{2}$ Oxisorbs, ${ }^{3} 13 \mathrm{X}$ molecular sieves, ${ }^{4}$ and electropolished stainless steel tubing.

The TMAE ${ }^{5}$ is cleaned by the CRID group by (a) washing it with oxygen-free deionized water, (b) filtering it through silica gel and $3 \mathrm{~A}$ and $4 \mathrm{~A}$ molecular sieves, and (c) pumping [22] it at an elevated temperature $\left(-60^{\circ} \mathrm{C}\right)$ and at a pressure of a few Torr for 12-24 hours. Initial cleaning by a simple filtration did not yield consistent results, which shows again that even a simple distillation is a preferred method to filtration. This TMAE cleaning method was perfected over many years, and resulted in the production of consistently good quality TMAE by removal of various contaminants, such as TMO, which is water soluble (it has a $\sim 40 \mathrm{x}$ times higher electron capture rate than oxygen [23]). Before the TMAE is used in the TPC's, it is qualified in a small ionization chamber (ELM) [24]. Figure 7 shows an example of an electron lifetime before and after TMAE washing. One can see a clear improvement in TMAE purity when pumping is used compared to simple filtering. When the TMAE washing is finished, the electron lifetime is almost the same in the TMAE laden gas as in the carrier gas, in this case, $\mathrm{CH}_{4}$ [11].

However, it was sulfur, not TMAE, which was the biggest source of grief for long term operation of the CRID. Sulfur can exist in ethane as elemental sulfur, $\mathrm{CS}_{2}, \mathrm{H}_{2} \mathrm{~S}, \mathrm{SO}_{2}$ and $\mathrm{COS}$. Our initial 1991 ethane supply was contaminated at a level of only 1-3 ppm, but that was sufficient to completely plug the ethane pressure regulator after one year of operation. During the 1993 physics run, we tried using a nickel getter ${ }^{6}$ to remove this contaminant. This caused day-to-night variations in drift velocity, which could not be corrected by means of a simple pressure and tempcraturc correction. Instead, we had to rely on the UV fiber drift velocity off-line calibrations. There was also a small release of oxygen from the nickel getter over a period of time, which prompted us to introduce another Oxisorb cartridge on the gas pad. This experience illustrates that some very active substances used in modern cleaning filters, such as nickel, can give rise to very complicated chemistry. As a result, in 1994, we decided to buy sulfur-free ethane from Europe. The sulfur level was specified to be less than 1ppm. The 1994 and 1995 physics runs went without any problem. However, in the 1996 and 1997 runs, the same plug-up problems developed. For example, we saw elemental sulfur deposits in the gas regulator, and we also clearly recognized the characteristic smell of $\mathrm{SO}_{2}$. Fortunately, sulfur was always caught in the first Oxisorb and never got to the detector. However, it did cause several hard plugs in the high-pressure section of the plumbing, which is always a severe problem when running with TMAE. Until now, the "fix" to this problem was to avoid any cold spot in the high-pressure section of the gas tubing since the sulfur deposits are found near such cold spot.

\section{Gas Radiator}

Our gas radiator recirculation system operates as a heat engine (see Fig.8). The original plan assumed filtering, however, it was soon realized that a simple distillation and condensation cycle in our recirculation system helps to maintain the required purity. The returning gas mix from the vessel enters a tank kept at $-90^{\circ} \mathrm{C}$ where the $\mathrm{C}_{5} \mathrm{~F}_{12}$ liquefies, and the $\mathrm{N}_{2}$ gas is vented (this also serves to remove some impurities). The $\mathrm{C}_{5} \mathrm{~F}_{12}$ liquid is then evaporated again and mixed with new $\mathrm{N}_{2}$ gas. The overall flow generates one complete volume change every 10-11 hours. In parallel, the $\mathrm{C}_{5} \mathrm{~F}_{12}$ liquid is circulated through a system of silica gel, ${ }^{7}$ elemental copper (RIDOX) ${ }^{8}$ and Oxisorb filters. The idea is that the silica gel protects the other filters. ${ }^{9}$ Figure 9 shows a

\footnotetext{
1 Purchased from AlphaGaz Co. with purity specification: $99.995 \%$.

2 Mechanical Waferguard filter, 0.05 micron, Milipore Co., U.S.A.

3 Oxisorb made by Messer Griesheim GmbH, 4000 Dusseldorf, Germany.

4 13X Molecular Sieve made by Union Carbide Co., Danburg, CT 06817-0001, U.S.A.

5 TMAE stands for Tetrakis dimethylamino ethylene, and was purchased from RSA Co., U.S.A.

6 Nickel catalyst N1-0104T-1/8 made by Engelhard Co., Iselin, NJ 08830-0770, U.S.A.

7 Silica Gel Sorbead R was purchased from Adcoa Co., Gardena, CA 90247, U.S.A. 
good reproducibility of the transmission measurements using liquid samples from the $-80^{\circ} \mathrm{C}$ tank, taken during different periods. The CRID measurements are only slightly worse than the results from DELPHI [25].

We mix $\mathrm{C}_{5} \mathrm{~F}_{12}$ with $\mathrm{N}_{2}$ to be able to run the overall system at $35^{\circ} \mathrm{C}$, which is a requirement posed on us by the rest of the SLD. Any instability in the $87 \% \mathrm{C}_{5} \mathrm{~F}_{12}+13 \% \mathrm{~N}_{2}$ mix causes instability in the refractive index, and thus the Cherenkov angle. The gas radiator mixture in the vessel is continuously monitored by sonar [26]. Figure 10 shows the time history of the Cherenkov angles in the radiator obtained from off-line reconstruction, in comparison to the angles obtained from the sonar measurements.

\section{E. Liquid Radiator}

The $\mathrm{C}_{6} \mathrm{~F}_{14}$ liquid is initially de-oxygenated purging $\mathrm{N}_{2}$ gas through it. Filtering it through the Oxisorb then purifies the liquid. The recirculation flow rate is one radiator volume every two hours. The Oxisorb is changed every three months. In this system, the Oxisorb is not protected by silica gel. However, we do know that $3 \mathrm{M} \mathrm{Co}$. uses silica gel to clean the liquid before shipping, and our experience to date has been satisfactory. We have never experienced problems with improperly fluorinated $\mathrm{C}_{6} \mathrm{~F}_{14}$ molecules such as those experienced by the DELPHI group [25,27], which caused severe transmission problems in the early period of their running. However, Fig.11 shows that our simple filtering method is not equivalent to cleaning by fractional distillation, which DELPHI presently uses to separate $\mathrm{C}_{5} \mathrm{~F}_{12}$ and $\mathrm{C}_{6} \mathrm{~F}_{14}$ molecules, due to leaks between the liquid system and the gas radiator. Figure 11 also shows that by the time of the Bari RICH conference, both experiments had a similar transmission; at that time, DELPHI did not yet use the distillation process; after the process was introduced, one can see a steady improvement. One can also see that the 1986 DELPHI prototype had even worse transmission.

\section{G. Material Stability}

Generally, all materials exposed to TMAE are in excellent shape after $\sim 7$ years of SLD operation and 10 years after the initial construction. This includes hard epoxies (DP-190 or Epon826+Versamid140), Cu-Be electrodes of the, Viton O-rings ${ }^{10}$ used to $^{1}$ seal the MWPC detector to the TPC, TMAE bubblers, etc. This comes from visual inspection of both TPCs and the MWPC detectors, and also from the fact that the oxygen level in the return gas from all TPC's is the same as seven years ago, i.e., below $1 \mathrm{ppm}$.

However, we see clear signs that the $\mathrm{C}_{5} \mathrm{~F}_{12}$ molecule, which is a solvent, leaches a small amount of plasticizer from the RF gasket $^{11}$ used to seal the radiator vessels' sector covers. The plasticizer is deposited on the clamps located a few mm away. However, because we keep a slight positive pressure in the vessel ( $\sim 0.5$ Torr), this effect is believed not to be harmful to sensitive components within the vessel.

We do not see any obvious deterioration due to $\mathrm{C}_{6} \mathrm{~F}_{14}$. However, based on our lab tests, the liquid trays, which were built using G-10 panels and quartz windows, do change shape under gravity. It is possible that this effect results in poorer angular resolution than expected for Cherenkov rings in the liquid (we have to add $\sim 10 \mathrm{mrad}$ in quadrature with the resolution obtained in Monte Carlo simulation to reproduce the data).

\section{H. CRID Monitoring}

There are several levels at which the CRID system is monitored. The absolutely critical variables, such as TPC or gas radiator pressure, are tied to an automatic hardware control, which protects the basic integrity of the system by switching off the gas flow [28]. The next very important part of the safety system is the SIAM alarm system, which allows monitoring of about 80 variables. Some critical variables are connected to an auto-dial paging system, and some can cause an automatic TMAE

\footnotetext{
8 Elemental Copper is made by Engelhard Co., Elyria, OH 44035, U.S.A. (The process is called Q-5.)

9 The procedure recommended to us by $3 \mathrm{M}$ Co., St. Paul, Minn. 55144-1000, U.S.A.

10 Viton O-ring, Parker Co., Part number : V0747-75, Cure 4Q87.

11 "Elastomet" rubber gasket filled with 5056 aluminum wire made by Nova-tronix Inc., Santa Clara, CA 95054, U.S.A.
} 
bubbler bypass; without the SIAM system, the CRID would not have been successful. Another important feature of the CRID is the slow, self-monitoring of each TPC. For example, the Cherenkov angle distribution is plotted at the end of each four-hour running period [see Figures 12(a)-(b)]; in a way, this is the final arbiter of performance. In addition, there is an extensive on-line monitoring system [29], which gives quantities such as the electron lifetime in selected TPCs, etc.

\section{Simple Estimate of $N_{o}$}

It is useful to define a "starting efficiency," which can then serve as a limit on how well one can do in the best possible circumstance, and is defined by the TMAE quantum efficiency and basic material transmissions. For liquid rings, the "starting efficiency" is defined by the wavelength dependent transmission through quartz, $\mathrm{C}_{6} \mathrm{~F}_{14}$ liquid, and the TMAE quantum efficiency. For gas rings, the "starting efficiency" is defined by the transmission through the quartz window, $87 \% \mathrm{C}_{5} \mathrm{~F}_{12}+13 \% \mathrm{~N}_{2}$ gas, the mirror reflectivity, and the TMAE quantum efficiency. There are many corrections, which degrade the "starting efficiency." They are derived from a collection of efficiencies, such as those measured during the construction period (transmission of quartz, mirror reflectivity, and TMAE quantum efficiency [30]), and those measured during the run (MWPC chamber efficiency, UV transmission of fluorocarbons, oxygen, and water effects), together, with estimates of other factors, such as the absorption by the field cage wires, electron lifetime, TPC gaps, TMAE absorption length, etc. We call the degraded efficiency a "final efficiency." The "starting" and "final" efficiencies are shown in Figs. 13 and 14. Table 1 lists estimates of the total degradation factors between the "starting efficiency" and the "final efficiency." We see about 10 photoelectrons per gas ring, and 16-17 photoelectrons per liquid ring for di-muons [21], i.e., close to expectation for simple events-see Table 1.

The above mentioned method does not include effects such as those due to the confusion in the middle of jets (the cross-talk and amplifier recovery cleaning cuts, and small electron losses due to the Lorentz angle in the liquid rings). Therefore, it is expected to be useful only for simple events, such as di-muons, where we observe 16-17 photoelectrons per full liquid ring, and $\sim 10$ per gas ring [29]; this is consistent with the simple calculation at the $10 \%$ level. Notice that we could increase the total number of photoelectrons per liquid ring by $2-3$ if we were to achieve DELPHI's $\mathrm{C}_{6} \mathrm{~F}_{14}$ purity.

\section{J. Angular Resolution}

Figure 15(a) shows the Cherenkov gas ring angular resolution for di-muons [21]. This particular resolution is obtained by making circle fits to gas rings, and represents the "internal resolution" (3.6mrad) of the CRID; it indicates that the TPCs are reconstructing data with a resolution close to design value. Figure 15 (b) shows the same for full liquid rings close to a $0^{\circ}$ dip angle. The expected resolution is about $12 \mathrm{mrad}$. This means that we have to add about $\sim 10 \mathrm{mrad}$ in quadrature to explain the data. The $\sim 10 \mathrm{mrad}$ represents systematic errors due to misalignments, for example, distortions in liquid trays. Figure 15 (c) shows the resolution as a function of dip angle.

\section{CONCLUSIONS}

A major achievement of the first generation photon detectors, such as the CRID, was to convince the many skeptics in the high energy physics community that the RICH concept can be implemented to yield useful physics. Today, nobody doubts that RICH is a useful concept. 


\section{REFERENCES}

[1] D. Muller, talk presented at this conference, RICH 98, Ein-Gedi, Israel, November 15, 1998.

[2] J. Sequinot and T. Ypsilantis, Nucl. Instr. Methods, 142 (1977) 377.

[3] DELPHI Proposal, LEPC 83-3 and LEPC 84-16, and Nucl. Instr. Methods, A343 (1994) 68.

[4] D.W.G.S. Leith, Nucl. Instr. Methods, A265 (1988) 120.

[5] K. Abe et al., "Fifth Int. Conference on Instrumentation for Colliding Beam Physics, Novosibirsk, Russia, March 15, 1990.

[6] K. Abe et al., Nucl. Instr. Methods, A343 (1994) 74.

[7] K. Abe et al., Nucl. Instr. Methods, A300 (1991) 501.

[8] S.Dasu, P.Rensing and J.Va'vra, CRID notc 73, July 20, 1994, and CRID note 72, April 9, 1993

[9] D. Aston et al., Nucl. Instr. Methods, A283 (1989) 590.

[10] J. Va'vra, CRID note 50, August 4, 1988.

[11] J. Va'vra, CRID note 89, January 28, 1997.

[12] F. Suekane and Y. Iwasaki, CRID note 79, March 24, 1993.

[13] Author's calculation using a code written by T.Ypsilantis.

[14] J. Va'vra, IEEE Trans. Nucl. Sci., NS-34, p. 486, Feb. 1987; CRID Note 36, 1987; and IEEE Trans. Nucl. Sci., NS-35, p. 487, Feb. 1988.

[15] J.Va'vra, Nucl. Instr. Methods, A387 (1997) 183.

[16] L. Malter, Phys. Rev. 1(1936)48-58, and Guentherschultze, Z. Phys. 86 (1933) 778.

[17] J. Va'vra, Nucl. Instr. Methods, A367 (1995) 353.

[18] E. Spencer et al., IEEE Trans. Nucl. Sci., NS-35, p. 231, Feb. 1988; P. Antilogus et al., SLAC-PUB-5120, Oct. 1990;

K. Abe et al., SLAC-PUB-5679, Nov. 1991.

[19] P. Rensing, Ph.D. thesis, SLAC Report 421, Aug. 1993.

[20] J.Va'vra, CRID note 77, February 3, 1993.

[21] K. Abe et al., IEEE Trans. Nucl. Sci., NS-40, No.4, p. 593, June 1993.

[22] D. Anderson, IEEE Trans. Nucl. Sci., NS-28, p. 842, Feb 1981.

[23] R.T. Rewick, T. Weber, M. Cavalli-Sforza, M.L. Schumacher, and S. Shapiro, Anal. Chem. 60 (1989) 2095.

[24] M. Cavalli-Sforza, CRID Note 15, May 1986.

[25] G. Lenzen, private communication, and E. Schyns, Ph.D. thesis, WUB-DIS-96-22, Wuppertal, Germany, 1997.

[26] G. Hallewell et al., Nucl. Instr. Methods, A264 (1988) 219; H. Kawahara and J.Va'vra, CRID note 78, March 18, 1993.

[27] S. Ilie and G. Lenzen, DELPHI 93-33 RICH 54.

[28] P. Antilogus et al., Nucl. Instr. Methods, A293 (1990) 136.

[29] T. J. Pavel, Ph.D. Thesis, SLAC Report 491, Aug.1997.

[30] R.A. Holroyd, J.M. Preses, C.L. Woody, and R.A. Johnson, Nucl. Instr. Methods, A261 (1987) 446.

Table 1. CRID final performance for gas and liquid rings for simple events such as di-muons [11].

\begin{tabular}{|l|c|c|}
\hline Parameter & Gas rings & Liquid rings \\
\hline $\begin{array}{l}\text { Total fraction of photoelectrons contributing to the } \\
\text { "final efficiency" compared to the "starting efficiency" }\end{array}$ & 0.70 & 0.53 \\
\hline Mean photon energy [eV] & 6.70 & 6.50 \\
\hline Mean refractive index & 1.001646 & 1.27202 \\
\hline Mean Cherenkov angle [mrad] ( $\beta=1$ particle) & 57.33 & 666.24 \\
\hline Calculated for radiator length [cm] & 45 & 1 \\
\hline $\mathbf{N}_{\mathrm{o}}\left[\mathrm{cm}^{-1}\right]$ & 80 & 42 \\
\hline Expected average number of photoelectrons per full ring ( $\beta=1$ particle) [11] & $11-12$ & 16 \\
\hline Measured average number of photoelectrons per full ring (di-muons) [29] & 10 & $16-17$ \\
\hline
\end{tabular}




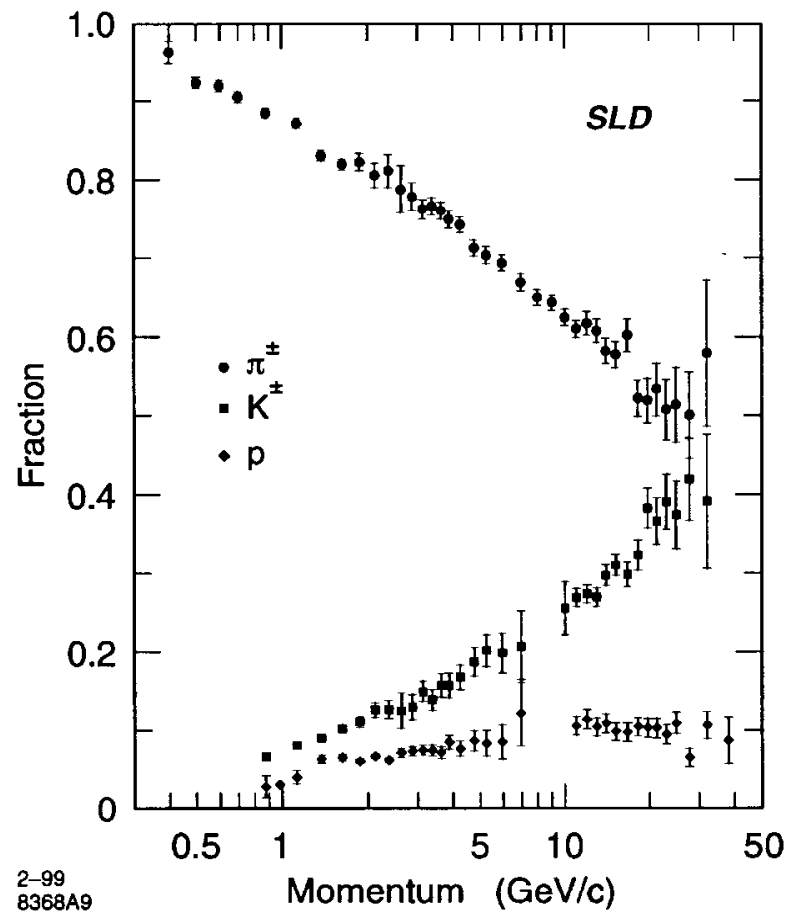

Fig. 1. Particle fractions from $Z^{\circ}$ decays as a function of momentum [1].

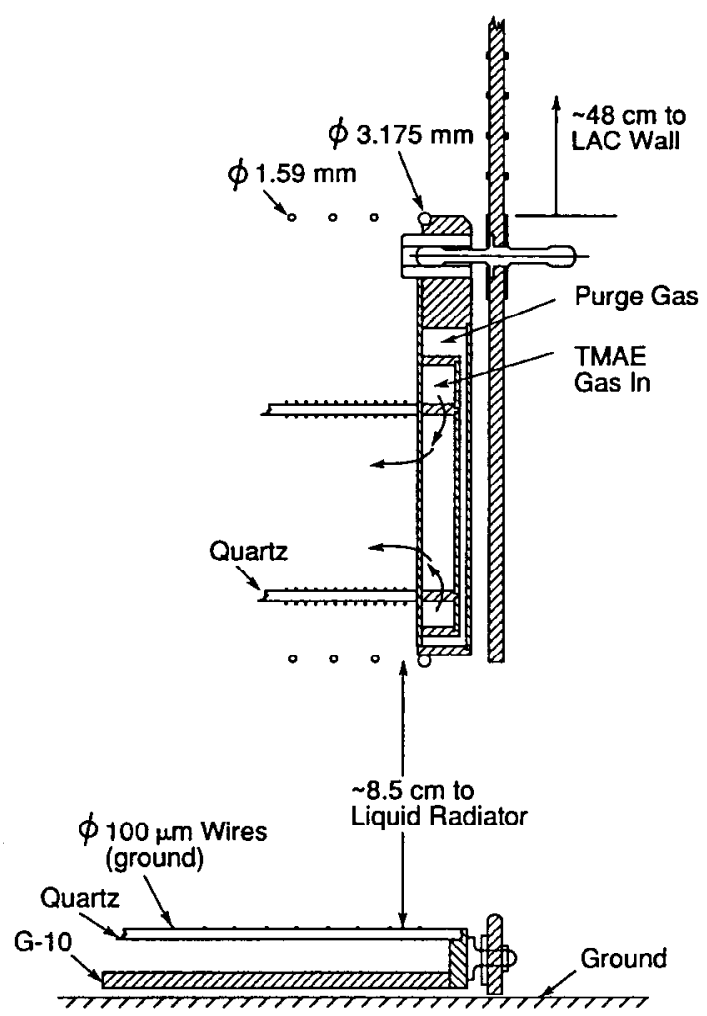

Fig. 2. The field cage on the drift box and liquid radiator near the high voltage end. 


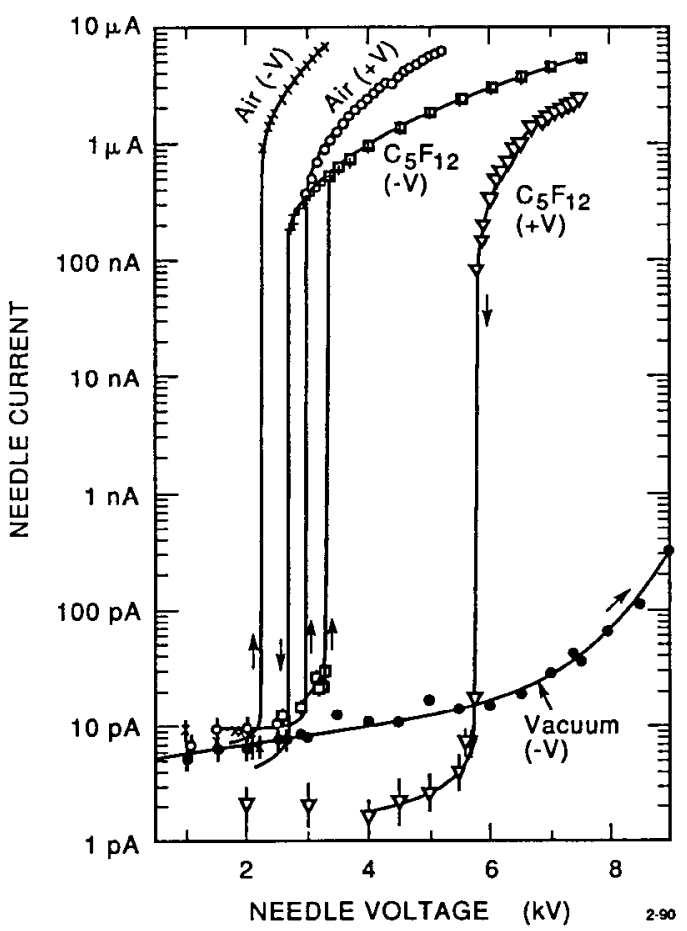

Fig. 3. Corona in $\mathrm{C}_{5} \mathrm{~F}_{12}$ gas compared to air in a geometry employing a needle as a cathode and a flat electrode as anode [8].

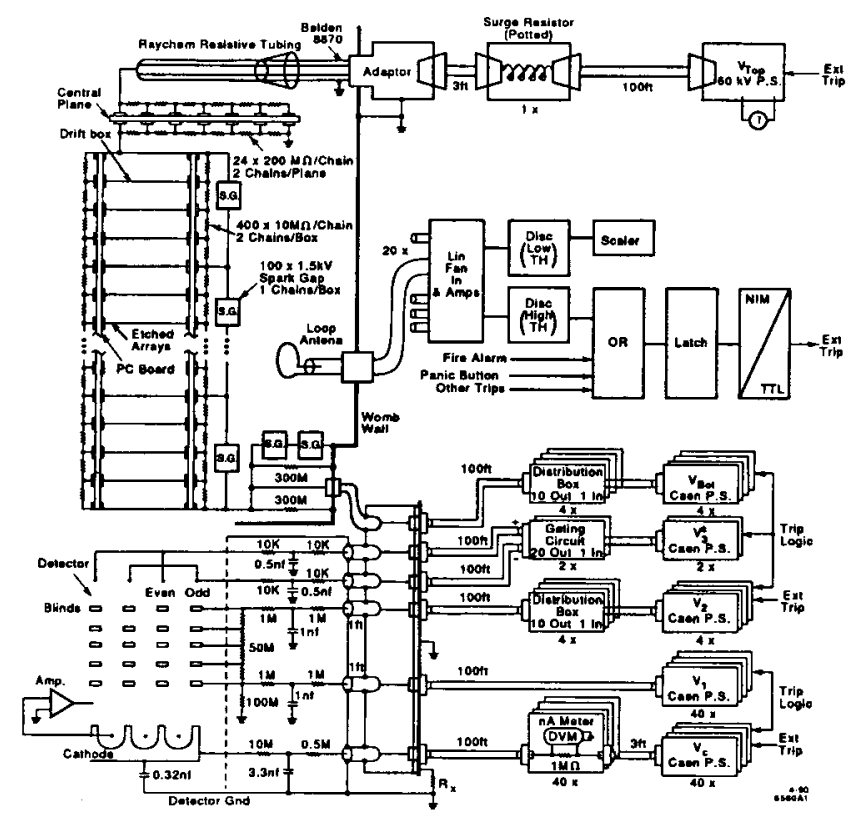

Fig. 4. CRID high voltage control system [9]. 


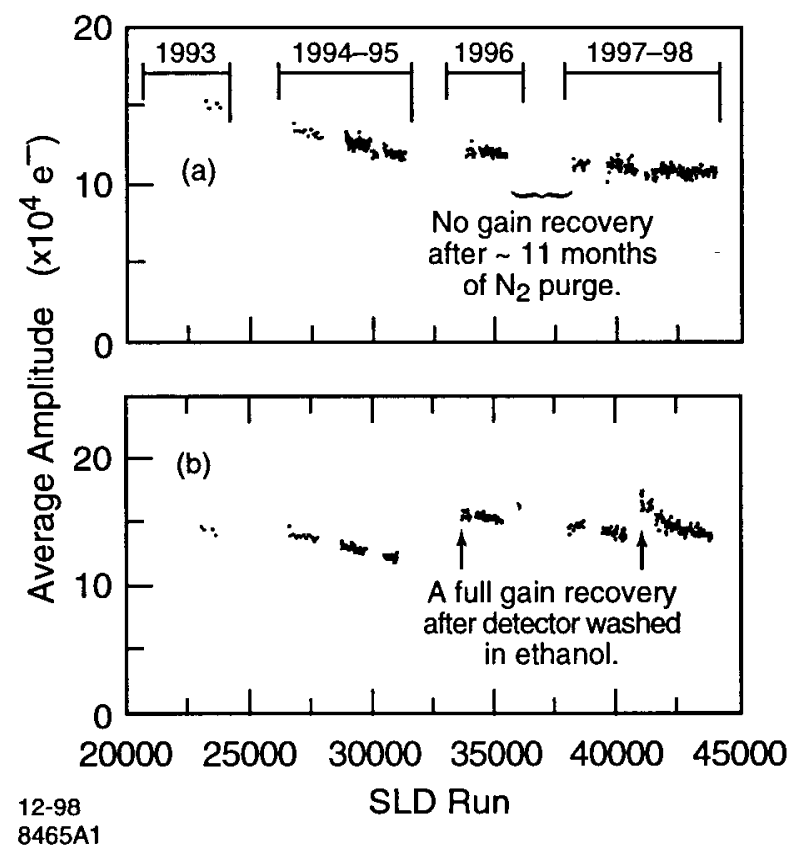

Fig. 5. Wire ageing in the SLD CRID between 1993 and 1998 with: (a) detector which was never removed, and (b) detector which was removed after the 1995 run and washed in ethanol.
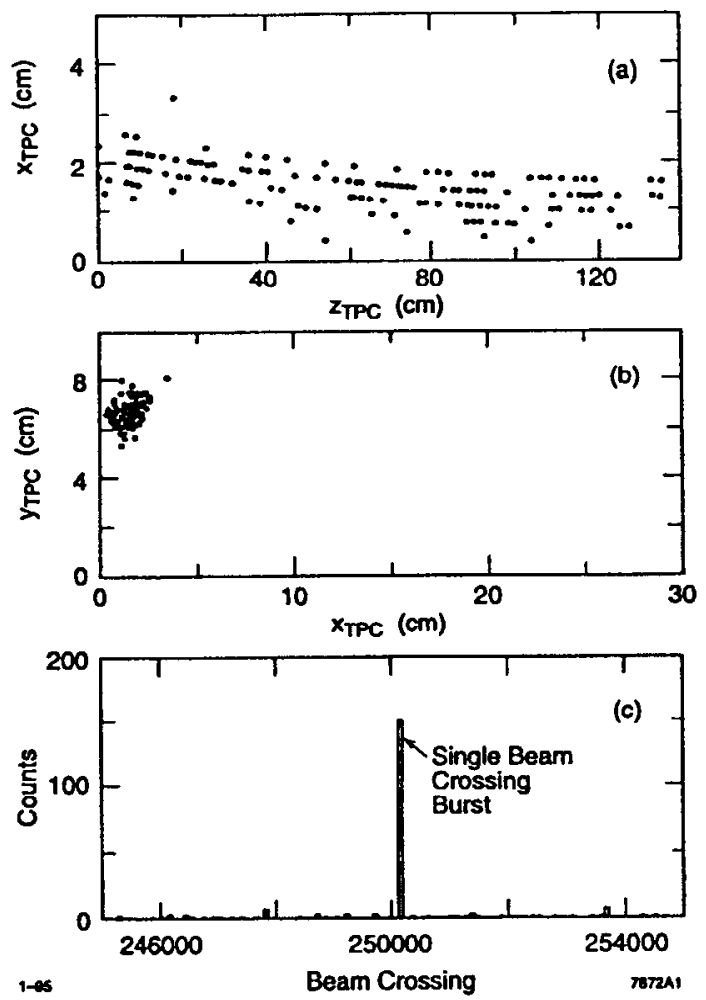

Fig. 6. Demonstration that the Malter effect can "manifest" itself also as a sporadic emission of charge in a form of single electrons. This was found because the CRID TPC can image not only physics events but also its internal behavior with excellent 3-dimensional reconstruction capability $\left(\mathrm{x}_{\mathrm{TPC}}, \mathrm{y}_{\mathrm{TPC}}, \mathbf{z}_{\mathrm{TPC}}\right.$ ), single electron sensitivity and time reconstruction [beam crossing corresponds to a fiber trigger in this case; the bursts is extinguished before the next trigger occurs $(<0.5 \mathrm{~s})]$. 


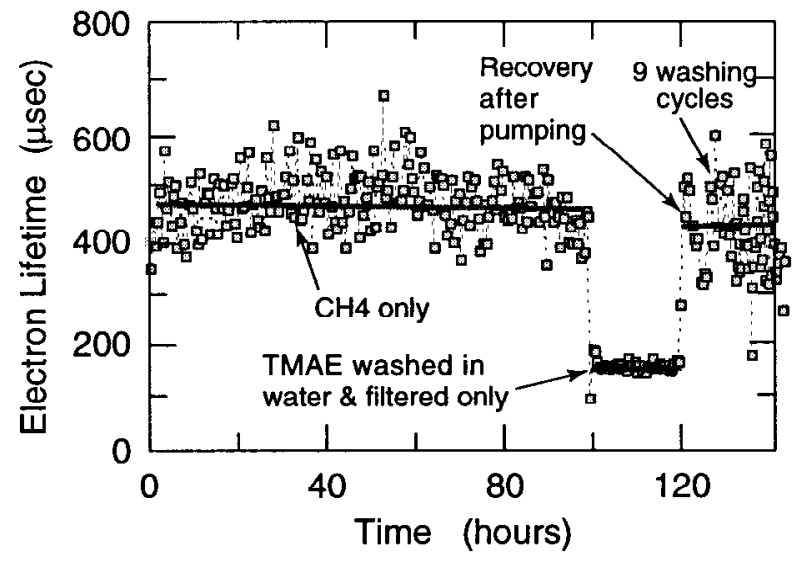

Fig. 7. TMAE washing: (a) measured electron lifetime with $\mathrm{CH}_{4}$ only, (b) with $\mathrm{CH}_{4}+\mathrm{TMAE}$ where TMAE was just washed in water and filtered, (c) as (b) but also pumped for 12-24 hours at a pressure of a few Torr at $60^{\circ} \mathrm{C}$ [11].

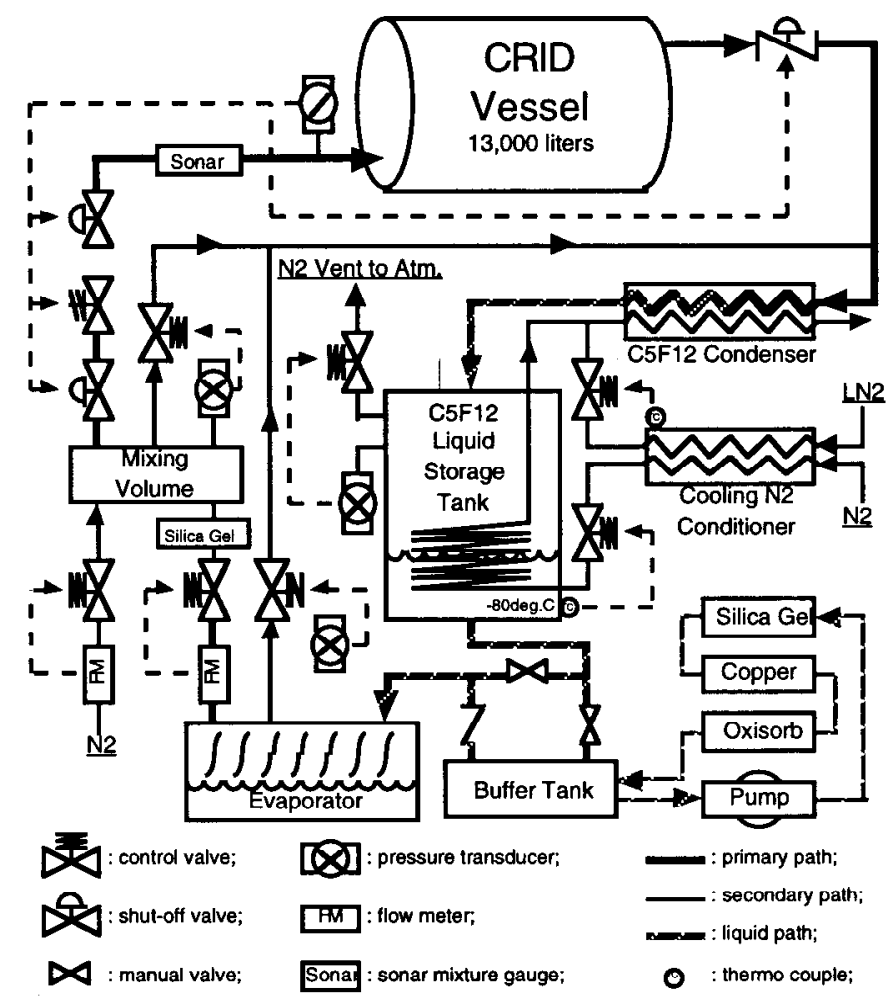

Fig. 8. Gas radiator purification system. 


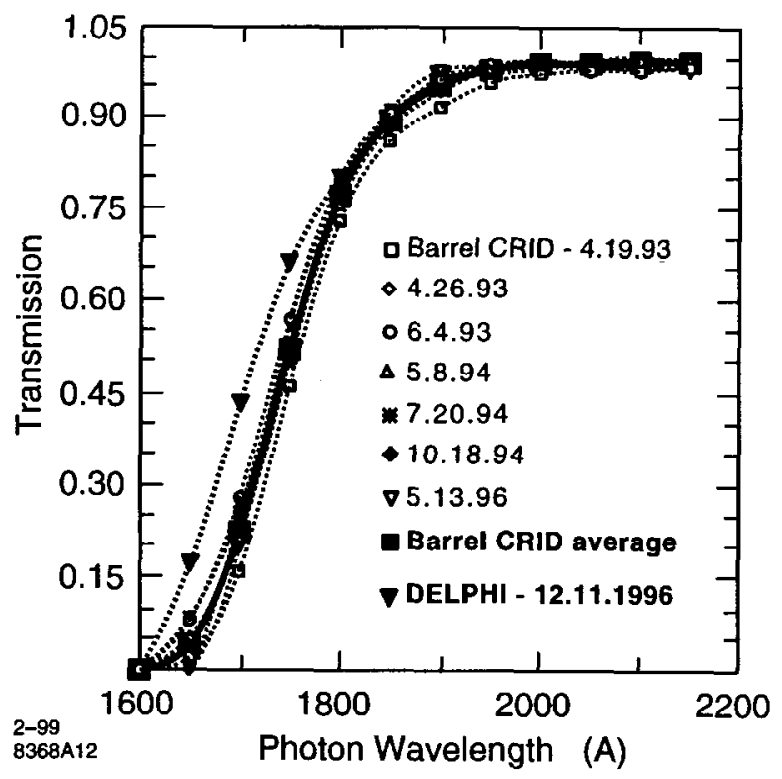

Fig. 9. The transmission of $\mathrm{C}_{5} \mathrm{~F}_{12}$ liquid taken from the $-80^{\circ} \mathrm{C}$ tank and measured in a $1 \mathrm{~cm}$ thick cell. Six SLD measurements from different periods, and their average, are compared to the current DELPHI results [11].

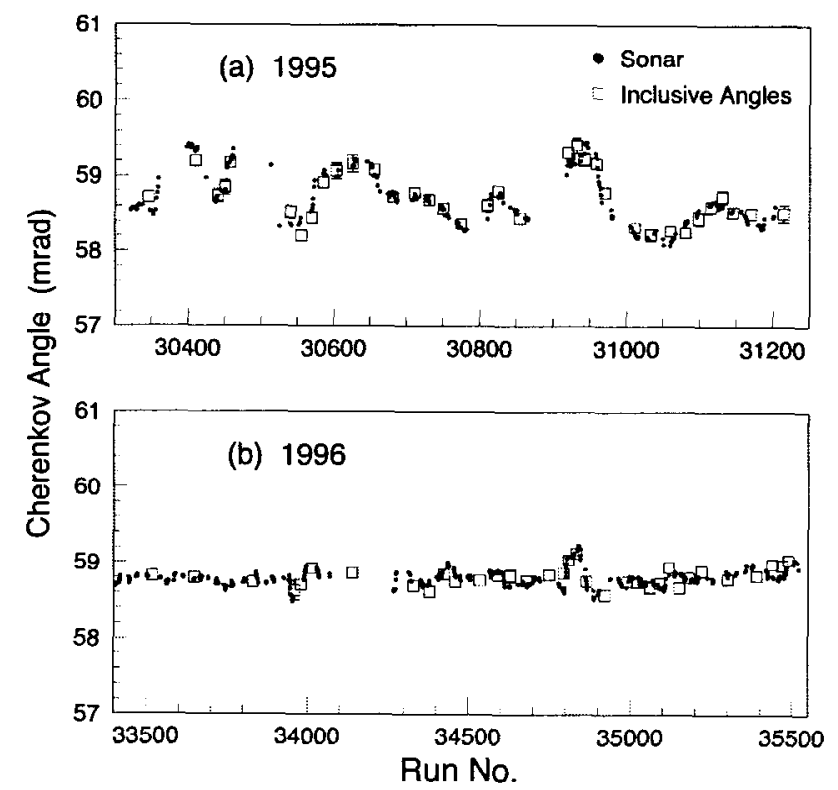

Fig. 10. The $\beta \sim 1$ Cherenkov angle measured from ring reconstruction (squares) compared to the value expected from the gas index measured by sound velocity in the CRID vessel and corrected for atmospheric pressure (dots). 


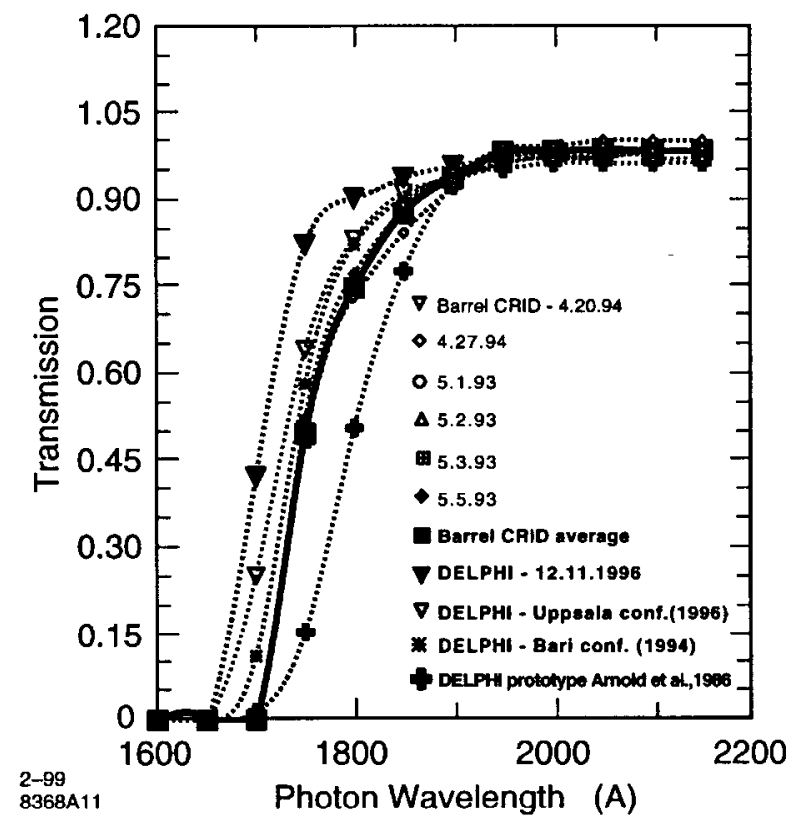

Fig. 11. The transmission of $\mathrm{C}_{6} \mathrm{~F}_{14}$ liquid measured in a $1 \mathrm{~cm}$ thick cell. Six SLD measurements from different periods, and their avcrage, are compared to the current DELPHI and older DELPHI prototype results [11].
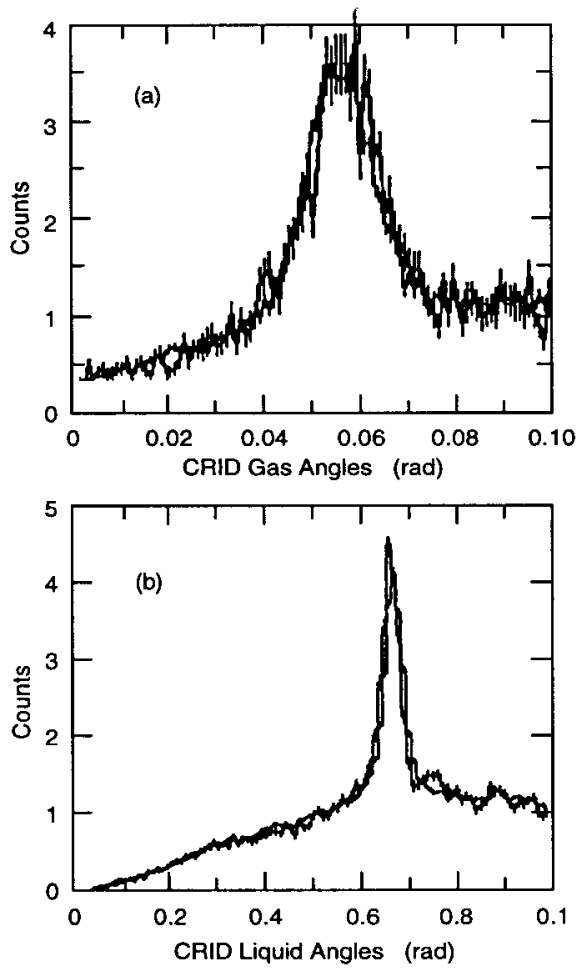

Fig. 12. (a)-(b) On-line monitoring of the Cherenkov angles at the end of each run ( $\sim 4$ hour running period). 


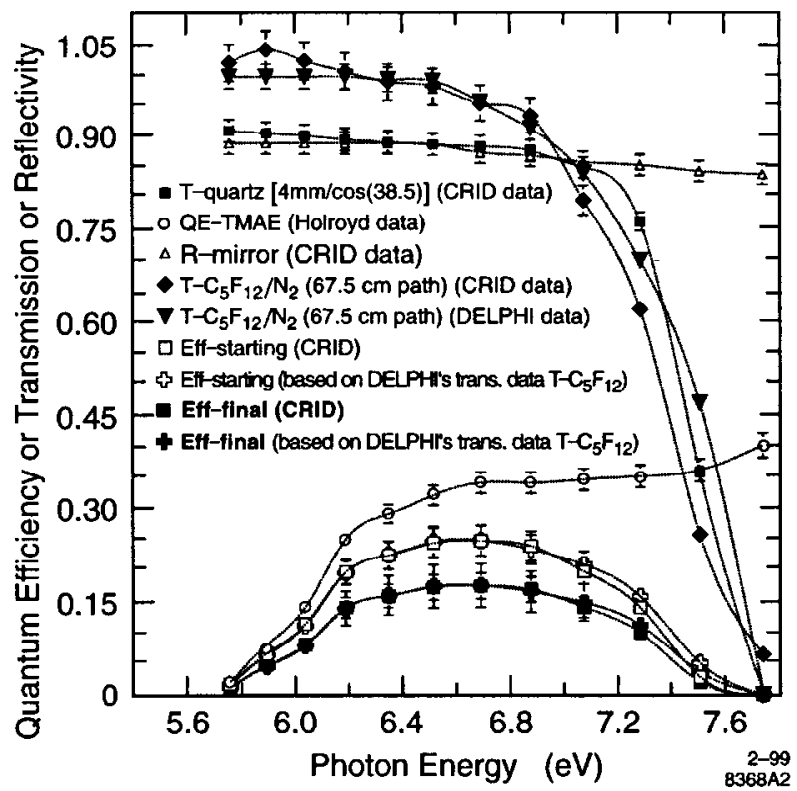

Fig. 13. Barrcl CRID gas rings with the $C_{5} F_{12} / N_{2}$ radiator: transmissions for quartz $(4 \mathrm{~mm})$ and the $87 \% \mathrm{C}_{5} \mathrm{~F}_{12}+13 \% \mathrm{~N}_{2}$ gas radiator [for an average photon path $\sim 1.5 \times 45 \mathrm{~cm}$ ], reflectivity of mirrors, TMAE quantum efficiency (Holroyd), and the "starting" and "final" efficiencies [11].

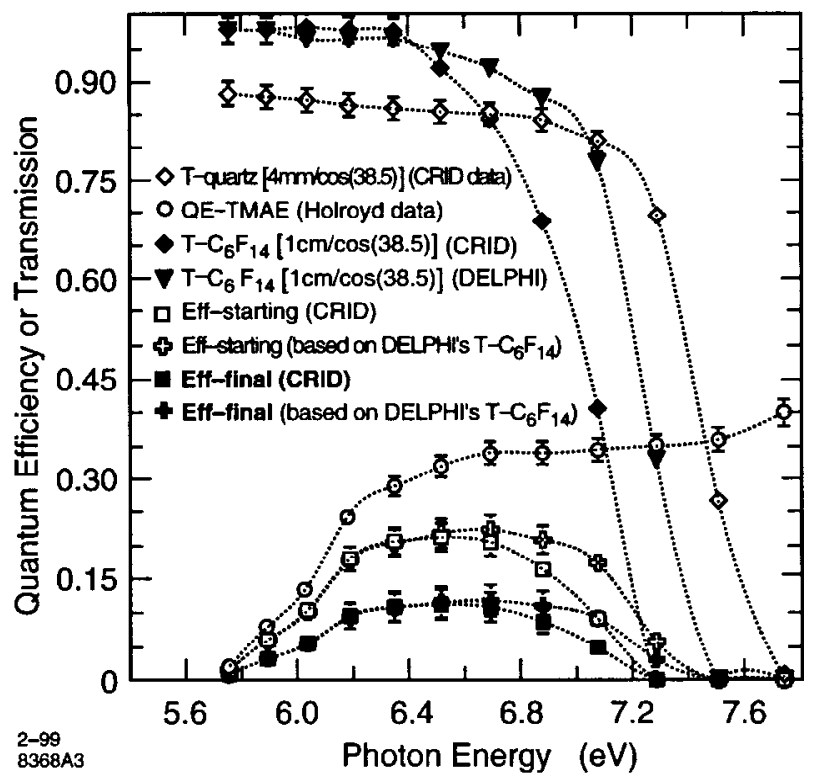

Fig. 14. Barrel CRID liquid rings: transmission for quartz $(4 \mathrm{~mm})$ and the $\mathrm{C}_{6} \mathrm{~F}_{14}$ liquid radiator $(1 \mathrm{~cm})$, TMAE quantum efficiency (Holroyd), and the "starting" and "final" efficiencies [11]. 

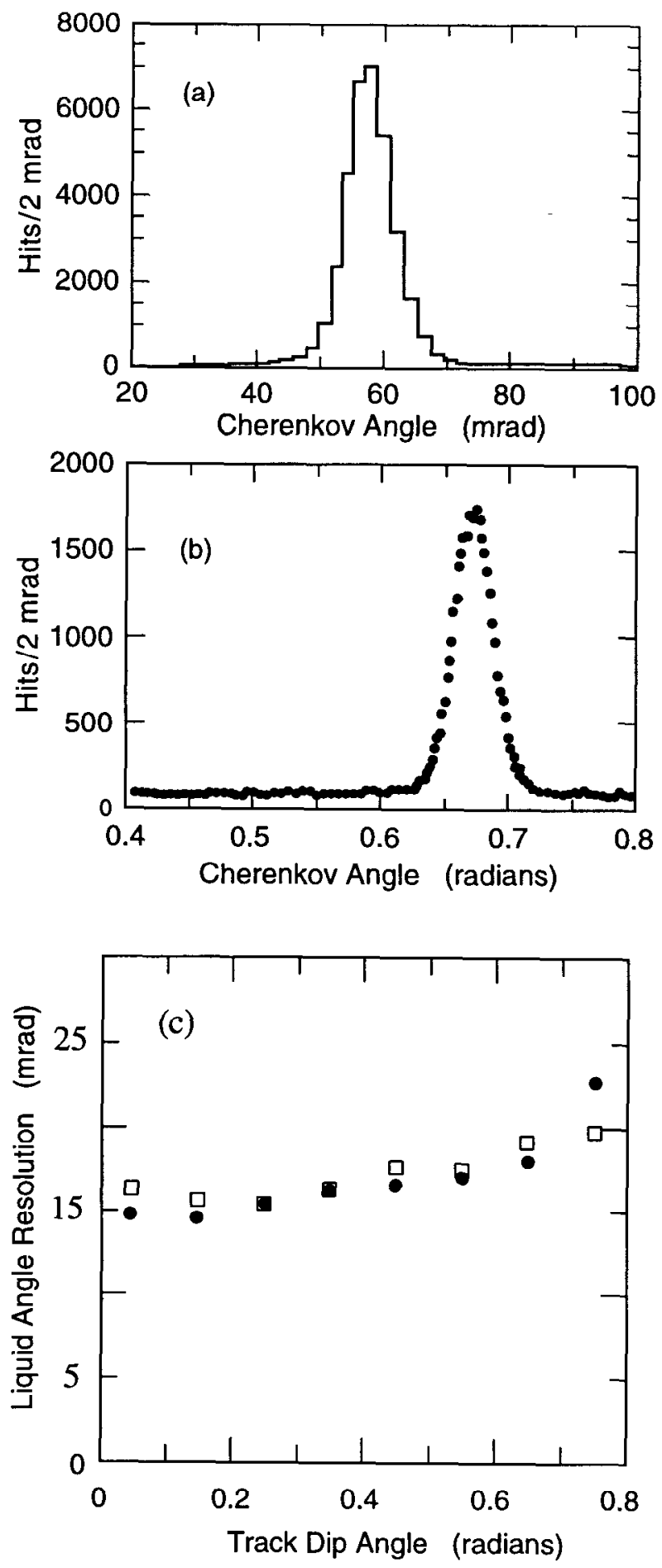

Fig.15 Cherenkov angle resolution in $\mathrm{e}^{+} \mathrm{e}^{-} \rightarrow \mu^{+} \mu^{-}$events for: (a) gas rings ( $3.6 \mathrm{mrad}$; residuals from the fitted circle to show the internal TPC resolution), (b) liquid rings ( $\sim 15 \mathrm{mrad}$; inclusive resolution) at $0^{\circ}$ dip angle, and (c) liquid rings as a function of dip angle (squares are from hadronic events, while dots are from $\mathrm{e}^{+} \mathrm{e}^{-} \rightarrow \mu^{+} \mu^{-}$and $\mathrm{e}^{+} \mathrm{e}^{-} \rightarrow \mathrm{e}^{+} \mathrm{e}^{-}$events) [21]. 